\title{
Localized recombination drives diversification of killing spectra for phage-derived syringacins
}

\author{
David A. Baltrus $\mathbb{B}^{1,2} \cdot$ Meara Clark ${ }^{1}$ Caitlin Smith ${ }^{1} \cdot$ Kevin L. Hockett $\mathbb{B}^{3,4}$
}

Received: 12 January 2018 / Revised: 16 May 2018 / Accepted: 6 July 2018 / Published online: 31 August 2018

(c) The Author(s) 2018. This article is published with open access

\begin{abstract}
To better understand the potential for antagonistic interactions between members of the same bacterial species, we have surveyed bacteriocin killing activity across a diverse suite of strains of the phytopathogen Pseudomonas syringae. Our data demonstrate that killing activity from phage-derived bacteriocins of $P$. syringae (R-type syringacins) is widespread. Despite a high overall diversity of bacteriocin activity, strains can broadly be classified into five main killing types and two main sensitivity types. Furthermore, we show that killing activity switches frequently between strains and that switches correlate with localized recombination of two genes that together encode the proteins that specify bacteriocin targeting. Lastly, we demonstrate that phage-derived bacteriocin killing activity can be swapped between strains simply through expression of these two genes in trans. Overall, our study characterizes extensive diversity of killing activity for phage-derived bacteriocins of $P$. syringae across strains and highlights the power of localized recombination to alter phenotypes that mediate strain interactions during evolution of natural populations and communities.
\end{abstract}

\section{Introduction}

Microbiome composition is dynamic through both space and time, but many questions remain concerning how specific factors shape these communities especially in the context of phytobiomes. Interactions mediated by the production of and sensitivity to molecules made by members of the community are known to play an important role in structuring microbiomes [1, 2]. Heightened appreciation for the influential role of microbes across of a suite of environments has driven new interest in developing and designing the strain specificity of these antimicrobials as a

$\triangle$ David A. Baltrus

baltrus@email.arizona.edu

1 School of Plant Sciences, University of Arizona, Tucson, AZ 85721, USA

2 School of Animal and Comparative Biomedical Sciences, University of Arizona, Tucson, AZ 85721, USA

3 Department of Plant Pathology and Environmental Microbiology, The Pennsylvania State University, University Park, PA 16802, USA

4 The Huck Institutes of the Life Sciences, The Pennsylvania State University, University Park, PA 16802, USA means to precisely engineer microbiomes [3, 4]. Given the potential for rapid evolution of pathways underlying these interactions, they may also be particular hotbeds for coevolution within microbiomes [5-7]. A more thorough understanding of underlying patterns and of the genetic basis of interactions between microbes will therefore shed new light on mechanisms that structure ecological and evolutionary dynamics across natural microbial communities and may enable development of targeted therapies to manipulate microbial composition across hosts and environments.

The best-characterized, and perhaps best-known, means to alter microbial community composition involves the use of broad spectrum antibiotics targeting physiological processes conserved across diverse bacterial taxa. However, lowered efficacy of broad spectrum antibiotics due to the evolution of resistance coupled with the realization that their use can lead to detrimental off-target effects on beneficial microbes has created new research momentum to identify and characterize new types of intermicrobial interactions that possess higher specificity [8-11]. Bacteriocins are molecules produced by bacterial cells that are largely considered to specifically target different strains of the same species or closely related species $[6,9]$. In contrast to relatively indiscriminate activity of some broad spectrum antibiotics, bacteriocin targeting is mediated through a 
requirement for interactions with particular receptors on target cells before antimicrobial functions take place $[6,12]$. The precision of bacteriocin killing activity also suggests that they are used in nature as a means to outcompete microbes that occupy similar niches, and thus characterization of bacteriocin activity and sensitivity from a diverse collection of strains could inform previously unrecognized ecological patterns [13]. Perhaps most relevant for their therapeutic use, the relatively specific killing spectrum of bacteriocins could provide a powerful means to precisely engineer microbiomes while avoiding off-target effects seen with other treatments $[4,9]$.

We have recently characterized a class of bacteriophage tail-derived bacteriocins produced by the plant pathogen Pseudomonas syringae, termed R-type syringacins [14]. These compounds are a member of a broader class of molecules, called tailocins, the production of which is controlled by bacteria but which are evolutionarily derived from coopted prophage $[15,16]$. Tailocins related to contractile tailed phages (Myoviridae) are categorized by the presence of genes encoding phage tail proteins (sheath, core, and baseplate constituents) and oftentimes the loss of genes encoding other structural or enzymatic proteins required for the production of active phage [16-19]. Production of phage-derived bacteriocins is often induced by the SOS response and therefore is triggered by exposure to DNA-damaging agents such as mitomycin $\mathrm{C}$. The specific regulatory components underlying the production of these particles has only been examined in Pseudomonas aerugi$n o s a$, where a protein with features characteristic of a LexAlike repressor, PrtR, is inactivated by RecA following DNA damage [20]. Once expressed, proteins are assembled into a structure that resembles a headless phage with upwards of hundreds of molecules made per producing cell and are often released through lysis of the producer cell $[12,16]$. Killing by phage-derived bacteriocins is thought to take place through a mechanism involving depolarization of target cell membranes upon puncture with coopted phage tail structures [16]. While phage-derived syringacins appear to be mechanistically and evolutionarily similar to R-type pyocins produced by $P$. aeruginos $a$ and are indeed found at the same genomic context (in between genes involved in tryptophan biosynthesis), they have a different evolutionary history as they were derived from a different progenitor prophage [14]. Moreover, R-type pyocins have been demonstrated to use lipopolysaccharide (LPS) of Gramnegative cells as a binding site and we have recently demonstrated that mutations affecting LPS (such as loss of O-antigen) result in R-type syringacin resistance [21, 22]. Although bacteriocin activity has been characterized across subsets of $P$. syringae strains, and while it is likely that much of the reported activity was due to phage-derived syringacins, earlier experiments were limited in their ability to analyze the genetic basis of changes in killing spectra because genome sequences were not available [23, 24]. The relative ease of modern genome sequencing has enabled a more thorough understanding of genomic evolution and phylogenetic relationships between strains of $P$. syringae [25]. We therefore sought to take advantage of this wealth of genomic data to characterize bacteriocin killing spectra and sensitivity across a diverse range of $P$. syringae strains in order to gain insights into evolutionary patterns and the genetic basis of phenotypic diversity in bacteriocin activity across this species.

Here we report the characterization of killing and sensitivity spectra of bacteriocins across a diverse panel of strains classified as $P$. syringae sensu lato. We find that many of the strains can be broadly grouped into multiple classes for both killing activity and sensitivity and that membership across these groups is correlated. We further investigate the genetic basis of switches in killing activity for multiple strain pairs and find that such switches are likely due to localized recombination of a handful of genes in the R-type syringacin locus. Lastly, we confirm that ectopic expression of just two genes is sufficient to switch phage-derived bacteriocin targeting in at least one pair of closely related strains. Our results highlight the important role that recombination plays in shaping a trait that likely influences community composition for this cosmopolitan environmental bacterium and paves the way for future studies to investigate the ecological and evolutionary basis of bacteriocin production and sensitivity across $P$. syringae.

\section{Results}

\section{Phylogenetic biases in syringacin production and killing}

We have performed an all-by-all screen across a diverse array of $P$. syringae strains to investigate production of and sensitivity to antimicrobial compounds induced by DNAdamaging agents. We have further used polyethylene glycol (PEG) precipitation to select for the subset of these compounds likely to be phage tail-derived bacteriocins known as R-type syringacins. Upon inspection of the killing matrix (Fig. 1), it is apparent that, although many of these strains harbor a variety of bacteriocins besides R-type syringacins, the dominant killing activity across all strains precipitates with PEG and thus is likely a high molecular weight compound. Furthermore, we note that killing activity of this high molecular weight compound was usually associated with crisp borders in agar overlay assays, which is strongly suggestive that these compounds are truly R-type syringacins (data not shown). This result echoes a screen of bacteriocin killing capability performed on a more limited 

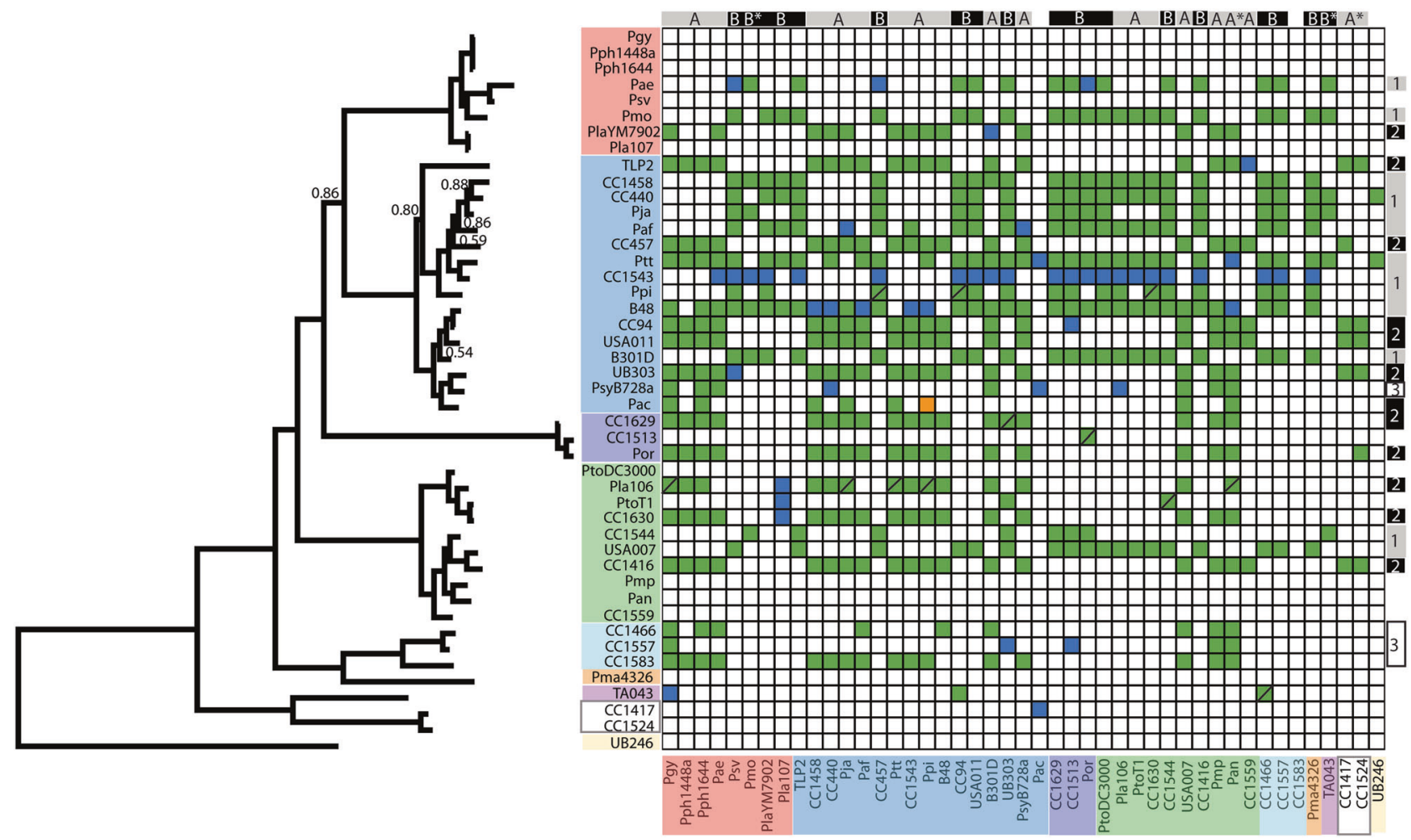

Fig. 1 Extensive R-type syringacin killing activity across diverse $P$. syringae strains. Strains are listed in both axes according to phylogenetic relationships. On the left of the figure is a phylogeny inferred from concatenated fragments of nucleotide sequences for five genes typically used in MLSA analysis of $P$. syringae $(g y r B$, rpoD, gapA, pgi, $a c n B)$. Posterior probabilities $<0.95$ are shown. Strain names are colored on both axes by their traditional placement into phylogroups. The $x$ axis of the matrix displays strain sensitivities to killings, while the $y$ axis displays strain killing activity. Colored boxes indicate killing activity according to overlay assays. Solid boxes indicate repeatable

collection of strains and points to an important role for Rtype syringacins in structuring communities of $P$. syringae [14].

We have ordered the killing matrix by phylogeny and colored strains according to phylogroup as described in ref. [26] so that proximity of producer and target strains along with coloration provides a readout on overall evolutionary similarity (Fig. 1). Closer inspection of the overall killing matrix in the context of phylogenetic relationships reveals numerous clear patterns as well as a handful of important yet nuanced results. The majority of strains exhibit killing activity consistent with phage-derived bacteriocins to at least one other strain in this screened collection (31/45, $68 \%$ ). In particular, strains within phylogroup II (14 of the 15 strains) seem to be exceptionally capable of killing other $P$. syringae strains within our collection, as almost all of the tested members can target at least one other screened strain. In no case did we find strains which produce molecules that possess killing activity against themselves. Furthermore, although they are the minority, multiple strains show no killing activity across multiple assays, whereas boxes with slashes indicate that killing activity existed in at least one assay. Blue boxes indicate killing activity only present in pure supernatants and thus consistent with diffusible bacteriocins, while green boxes indicate killing activity that was present in PEG-selected supernatants and is thus consistent with phage-derived bacteriocins. The orange box indicates killing activity that was repeatedly witnessed only after PEG precipitation. On the top of the matrix, strains have been grouped into sensitivity clusters, while on the right side of the matrix strains are grouped into killing clusters

killing activity against our strain collection and these strains generally clustered by phylogeny. For instance, two strains sampled from phylogroup IX (CC1417 and CC1524) do not show any killing activity consistent with R-type syringacins. Likewise, multiple closely related strains from phylogroup I (Pmp, Pan, CC1559) as well as phylogroup III (Pgy, Pph1448a, Pph1644) display no killing activity. In each of these cases, strains that lack killing activity form monophyletic groups compared to other strains within our screened collection.

\section{R-type syringacin killing activity can be broadly grouped into two high-activity clusters and at least five total groups}

As an additional step to characterize killing spectra, we used hierarchical clustering to group strains based on activity consistent with R-type syringacin killing activity (Fig. 2). According to groupings arising from this analysis, screened strains can be sorted into at least two deeply branching main 
Fig. 2 R-type syringacin killing and sensitivity frequently switch across strains. Strains were hierarchically clustered using the phenotypes of killing activity (left) and sensitivity (right) to PEG-selected supernatants. Strains are color coded by phylogroup as per Fig. 1. Killing spectra clusters $(1,2,3,4,5)$ are shown at nodes that differentiate these groups, and the number of other strains killed by each particular strain (without counting Pla107 due to inconsistent activity) is shown to the right of the strain name. On the right, strains are clustered by sensitivity to killing, with clusters denoted with letters $\left(\mathrm{a}, \mathrm{b}, \mathrm{A}^{*}, \mathrm{~B}^{*}\right)$

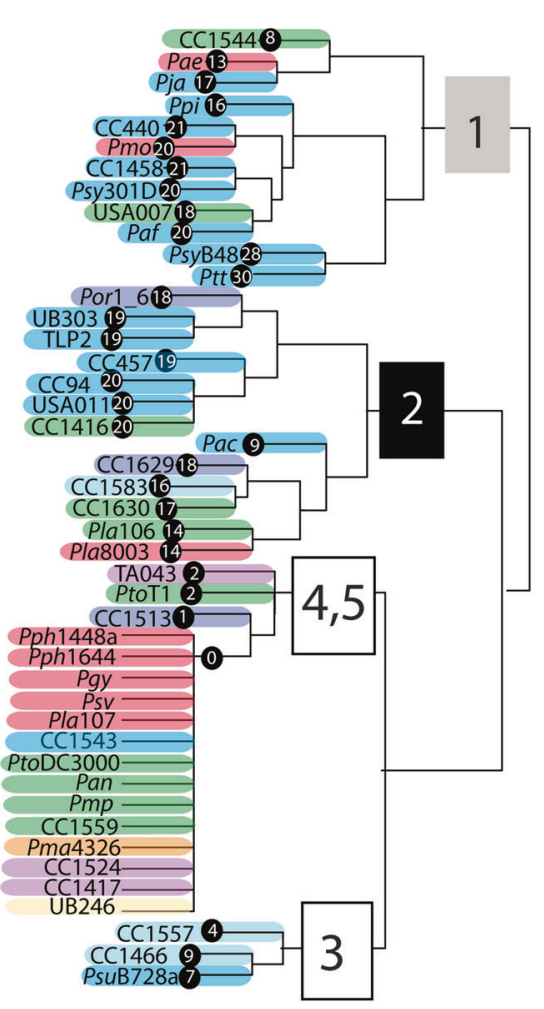

clusters (which we term clusters I and II) and at least five total groups. The two main clusters are dominated by strains with relatively broad killing spectrum (an average of $~ 19$ and $\sim 16$ strains targeted per cluster) but with little overlap between the strains targeted by each. A third group is nested within cluster II according to our analysis and contained killing activities that were relatively more selective (average of $~ 7$ ). The fourth group clusters closely with the third group mentioned above likely due to the relatively low number of targets but contains strains that have a minimal number (one or two) of targets. The fifth cluster is composed of strains with no observed killing activity.

It is also apparent that, even though many strains have broadly similar killing spectra, there are numerous instances where comparison of these spectra within clusters and groups reveals subtle differences (Figs. 1 and 2). For instance, PsyB48 and pathovar aptata (Ptt) have the broadest killing activities of any of the sampled strains, targeting 28 and 30 other strains, respectively. These strains can both kill the same 24 strains in our screen; however, PsyB48 can kill an additional 4 strains (USA007, Pja, Pmo, $P m p$ ) and $P t t$ can kill an additional 6 strains (UB246, Pph1644, CC440, Ppi, Paf, TA043). Likewise, while CC440 and CC1458 can both kill 21 strains, only 19 of these targets are shared by both strains as CC440 differentially targets TA043 and UB246 while CC1458 differentially targets Pmo and PsyB301D. The clustering of killing spectra between strains further demonstrates both that closely related strains (according to core genome sequences and phylogroups) can have different killing spectra and conversely that relatively distantly related strains have convergently evolved similar killing spectra.

\section{Rapid switches of syringamycin killing spectra across strains}

Comparison of the $P$. syringae phylogeny reported in Fig. 1 with R-type syringacin killing activity reported in Fig. 2 demonstrates that killing spectra evolve quite rapidly across strains. Although phylogenies built from conserved gene sequences recapitulate traditionally recognized phylogroup structures (see colored shading for phylogroups in Fig. 1), hierarchical clustering of killing spectra demonstrates that even closely related strains have dramatically divergent phage-derived syringacin targeting capabilities. Rapid diversification is most clearly seen by comparing coherent phylogenetic clustering of each phylogroup (represented by single blocks of color throughout the phylogeny) with the same colors represented in hierarchical clusters of activity. For example, compare strains PsyB301D and USA011, which are relatively closely related according to phylogenies built from housekeeping loci, and which target the same number of strains within our assays, but which have different killing spectra. 


\section{Diverse patterns of R-type syringacin sensitivity}

We have also performed hierarchical clustering on strain sensitivity to PEG-precipitated antimicrobial compounds (Fig. 2). It is also noteworthy that almost all of the P. syringae strains were able to be killed by at least one other strain within our collection, with the exception being CC1583 (we also found that Pac is killed by a diffusible compound but not an R-type syringacin). While, subjectively, there appears to be slightly more phylogenetic congruity overall when considering sensitivity compared to killing spectra (i.e., phylogroup IV strains cluster together for sensitivity but not for killing activity), it is clear that the results form strong parallels with patterns of killing activity described above. There are two main clusters of sensitivity classes, which we term here A and $\mathrm{B}$. We also note two subclusters (A2 and B2), which group separately from the main clusters. We refer to these as subclusters because their sensitivity spectra are subsets of those found within main clusters A and B. It is likely that A2 and B2 group together in this analysis and not with their related parent clusters, because they each are targeted by a small number of strains and clustering is based on phenotypic distance. Therefore, we believe that A2 and B2 cluster together because their patterns are dominated by the lack of activity compared to strains within $\mathrm{A}$ and $\mathrm{B}$, and differences in the small number of activities is not enough to override this signal.

There are multiple cases where sensitivity dramatically differs between closely related strains. For instance, strain CC457 from sensitivity group B is closely related to both $P a f$ and Ptt (which are both sensitivity group A). Likewise, USA007 is classified in sensitivity group A but is closely related to $\mathrm{CC} 1544$ and $\mathrm{CC} 1416$ from sensitivity group B. There are also numerous cases where relatively divergent strains have convergently evolved to have very similar sensitivities to syringacins. Only a small subset of strains screened herein appear to have the exact same sensitivity spectrum, and there are multiple instances where there are a small number of differences between strains with similar sensitivity spectrums.

\section{Localized recombination is apparent for a subset of R-type syringacin structural genes}

Genome sequences are available for each of the strains screened as part of this study (see https://doi.org/10.6084/ $\mathrm{m} 9$.figshare.5738523), and it is straightforward to comb through this genomic information in order to identify genotypic changes strongly correlated with switches in killing activity. Given that receptor-binding proteins $(\mathrm{Rbp})$ have been implicated in targeting of R-type pyocins, analysis of their sequences is a clear first step towards identifying determinants of R-type syringacin specificity. As a test of whether diversity in Rbps correlates with killing activity, we inferred phylogenetic relationships for these predicted receptor-binding loci and compared these trees to those inferred for multiple loci found in close genomic proximity and surrounding the Rbps in the genome (trpC/trpD as well as a predicted phage tail fiber protein and tail sheath). As highlighted by colors representative of phylogenetic placement of strains into phylogroups in Figs. 1 and 2, phylogenies built from sequences of loci bracketing the Rbp largely recapitulate that inferred from housekeeping gene sequences (Fig. 3a). Although the positions of a handful of nodes change, overall strains form coherent groups that match phylogroup assignments. Phylogenetic patterns found on either side of the Rbp are therefore strongly consistent with vertical inheritance of the R-type syringacin locus across the diversity of $P$. syringae. Strikingly, phylogenies built from the Rbp sequences display extensive differences from those inferred from sequences of conserved housekeeping genes, as well as those inferred from sequences for other phage-derived syringacin structural genes. For the receptor-binding locus, coloring of strain names and thus phylogroup placement of strains is mixed and not monophyletic. Moreover, phylogenies built from the sequences of Rbps form two clear clades and strain membership in these clades is consistent with groupings of killing activity shown in Fig. 2.

Differences in phylogenetic placement for the Rbp is clearly suggestive of extensive localized recombination involving Rbps compared to other surrounding loci that code for structural loci of R-type syringacins. To better gauge the breakpoints and extent of these recombination events, we mapped nucleotide diversity for genomic regions involving these genes from three pairs of strains that are closely related but cluster into different groups based on killing spectra (Fig. 3b; CC1544 and CC1416 from phylogroup I, PsyB728a and PsyB301D from phylogroup II, and Pmo and Pla7092 from phylogroup III). Conservation of nucleotides is shown by green blocks within Fig. 3b, while divergence is shown by yellow blocks. Surprisingly, the N-terminus of the Rbp is relatively highly conserved across strains but the levels of sequence conservation repeatedly shift from high to low at a point inside the predicted Rbp open reading frame. This N-terminal sequence therefore potentially acts as one anchor point for recombination events that diversify R-type syringacin killing activity. On the other side, there is no consistent pattern for conservation of nucleotide diversity downstream of the Rbp and chaperone which suggests that the other recombination breakpoints likely occur in different positions in each of these pairs. However, in each case, it appears as though the breakpoint is upstream of a protein annotated as a lysozyme-like protein that appears to be vertically inherited across $P$. syringae strains. Therefore, even though it is 
A)
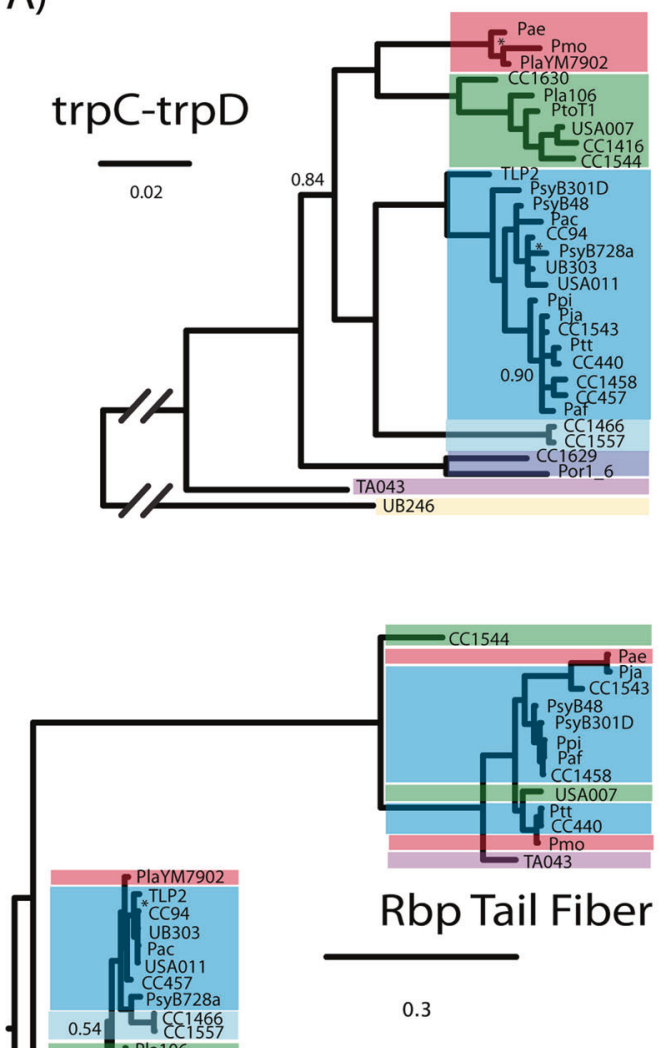

Tape Measure Protein-Sheath

Fig. 3 Localized recombination drives diversification of R-type syringacin killing activity. a Phylogenies are shown for genes found "upstream" (TrpC/TrpD) and "downstream" (Tape measure proteinTail sheath) of the receptor-binding protein for all strains that showed killing activity consistent with R-type syringacins within our assays (with the exception of $\mathrm{CC} 1583$, for which the Rbp sequence is truncated). A phylogeny is also shown for the receptor-binding protein itself. Strain names are color coded by phylogroups as shown in Fig. 1. Posterior probabilities $<0.95$ are noted with either the actual number or an asterisk (“*”) where space was limited. b Shown are nucleotide

B)

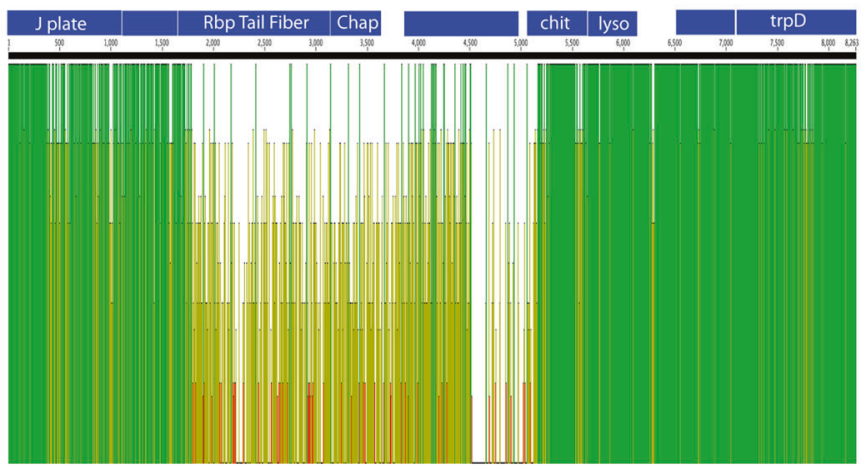

Pmo vs. PlaYM7902

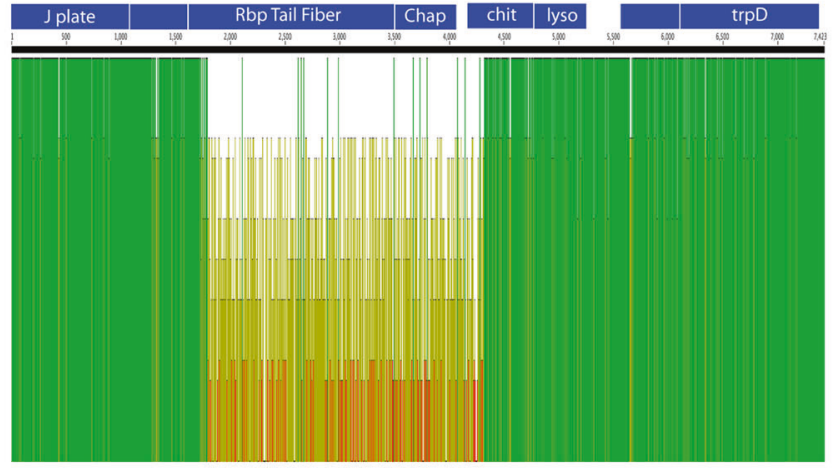

PsyB728a vs. PsyB301D

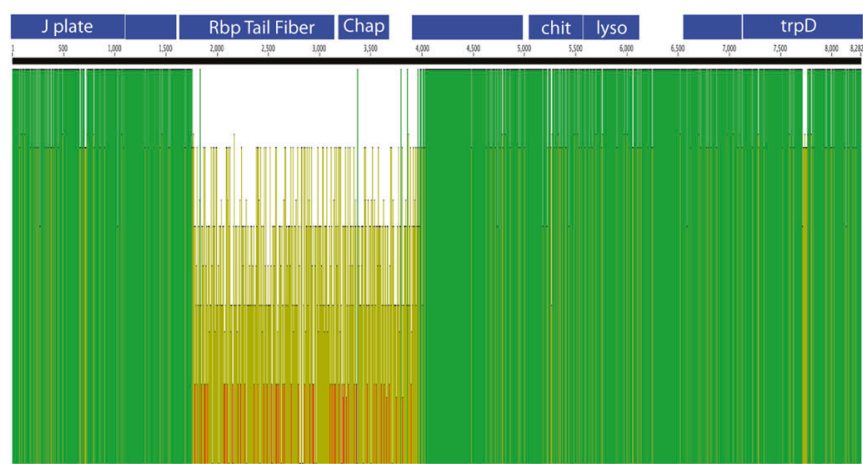

CC1544 vs. CC1416

sequence alignments for regions of the genome including the receptorbinding protein and chaperone pairs, as well as surrounding syringacin structural genes and $\operatorname{trp} D$. Predicted reading frames for genes found in this region are highlighted in blue at the top of each alignment. Green bars indicate high similarity in nucleotide sequence, whereas yellow bars indicate lower similarity. In each case, we find low nucleotide similarity for the area including most of the receptor-binding protein and chaperone and much higher similarity for regions upstream and downstream of these two genes 


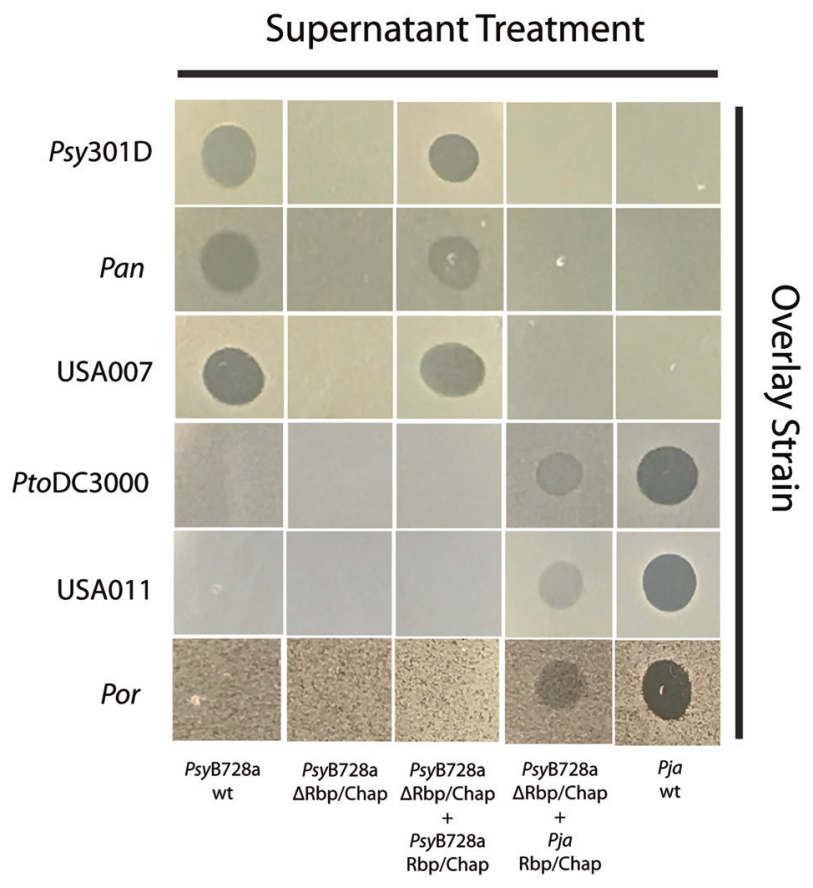

Fig. 4 Ectopic expression of the Rbp and chaperone is sufficient to retarget R-type syringacins. The results of overlays of six different strains and five different PEG-selected supernatants are shown. Overlay strains are shown on the $y$ axis, whereas supernatants are shown on the $x$ axis. Three strains are sensitive to killing by R-type syringacins produced by PsyB728a and not Pja (Psy301D, Pan, USA007), while three strains are sensitive to R-type syringacins produced by Pja and not PsyB728a (PtoDC3000, USA011, Por1_6). Killing activity is abolished in a mutant of $P s y \mathrm{~B} 728 \mathrm{a}$ where both the receptor-binding protein $(\mathrm{Rbp})$ and chaperone from the R-type syringacin has been deleted, and this mutant can be complemented by the expression of both these genes ectopically from a plasmid. Ectopic expression of the Rbp and chaperone from Pja in this PsyB728a $\Delta \mathrm{Rbp} /$ chaperone background retargets this strain so that the killing spectrum matches that of Pja

difficult to identify the ancestral state for any of these sequences, in each of the three independent pairwise comparisons the Rbp and chaperone have been cleanly switched through recombination and this switch is correlated with a complete overhaul of the killing spectra.

\section{Transfer of two genes is sufficient to change phage- derived syringacin killing spectrum}

To test whether recombination of the Rbp and chaperone alone are sufficient to retarget syringacins, we utilized a mutant strain of $P$. syringae pv. syringae $\mathrm{B} 728 \mathrm{a}$ (PsyB728a) in which both the $\mathrm{Rbp}$ and chaperone were previously deleted [14]. We have already demonstrated that we can complement phage-derived syringacin production and killing in this strain background by ectopically expressing the PsyB728a genes from a plasmid [14]. However, instead of complementing this strain with its native $\mathrm{Rbp}$ and chaperone, we instead chose to test whether killing activity of this strain could be complemented by both loci from a strain with a distinctly different killing spectrum $(P$. syringae pv. japonica, Pja). As shown in Fig. 4, not only can the Rbp and chaperone from Pja complement deletion of these genes from PsyB728a but the killing spectrum of this complemented strain is also consistent with the spectrum from Pja instead of PsyB728a. Therefore, horizontal transfer of both the Rbp and chaperone is sufficient to retarget the $P$. syringae $\mathrm{R}$-type syringacin.

\section{Discussion}

Phage-derived bacteriocins possess great potential as antimicrobials and may play significant roles in structuring bacterial communities under natural conditions $[6,7,15]$. Given the potential for strong selective pressures to diversify killing spectra, and likewise evolutionary pressure to gain resistance, bacteriocins are also likely to be hotspots for coevolutionary interactions between closely related strains. In order to shed light on evolutionary histories of phage-derived bacteriocins in the phytopathogen $P$. syringae (termed R-type syringacins), we performed a screen to measure the spectra of killing activity across a diverse collection of strains and have used genome sequence information to match phenotypic changes to underlying genetic differences.

\section{Phage-derived bacteriocins are the main intra- species killing activity for $P$. syringae}

Our results not only demonstrate that R-type syringacins are the dominant inducible bacteriocin produced across these strains but also highlight rapid evolutionary shifts in both syringacin target range as well as resistance. Broadly, we find that both R-type syringacin killing spectra and sensitivity to R-type syringacin killing across many strains can be grouped into main two clusters for the strains assayed (1 and 2 for killing, A and B for sensitivity). Moreover, membership in each cluster is highly correlated: Killing spectra cluster 1 is typically found within strains that are members of sensitivity cluster B and target strains from cluster A, while killing spectra cluster 2 is typically found within strains that are members of sensitivity cluster A and target strains from cluster B. There are two strains that do not fit cleanly into killing groups 1 or 2 but instead reflect a mix of both of these (PsyB48 and Ptt). Aside from strains that could not easily be grouped into killing and sensitivity clusters, there are three strains that seem to be exceptions to these correlations (Pmo, CC1544, PsyB728a). Notably, each of these strains is resistant to its own R-type syringacin even though they are sensitive to other tailocins from the 
same killing group. Our groupings differ from the previous groupings reported by Vidaver and Buckner [23, 27], and there are several possible explanations for these discrepancies. First, we clustered strains with similar, but not necessarily identical, killing spectra into the same killing group using a metric of phenotypic distance, whereas in the previous work subtle differences in killing spectra resulted in distinct bacteriocin-producer groupings. Second, our assays focused largely on R-type syringacin-mediated killing, while the previous work did not distinguish between Rtype and other syringacin activity. Finally, the previous work focused largely on a collection of strains belonging to pathovars syringae, glycinea, and phaseolicola (phylogroups II and III). Thus their collection could have potentially teased apart more nuanced interaction by deeper sampling within a narrower phylogenetic range of strains.

\section{Independent absence of R-type syringacin killing activity across $P$. syringae}

There are numerous instances where surveyed $P$. syringae strains have no R-type syringacin activity across this screened collection. It is likely that the "non-producer" strains are a group composed of strains with specific killing activities (but for which we have not included potential targets in our screen) as well as strains that are truly "nonproducers". This group could be split based on future studies, either by finding strains for which they can target or by genomic comparisons of syringacin structural genes combined with moving the Rbps to known producer strains to show that activity exists if the rest of the syringacin structural proteins are made. Further investigation suggests that, at least in a subset of cases, that losses of activity have independently occurred and are the product of different underlying genetic causes. Two strains from phylogroup IX show no activity consistent with R-type syringacins, and further inspection of their genome sequences suggests that they lack all of the genes known to be associated with $\mathrm{R}$-type syringacins. Indeed, there is only one gene predicted to be found between $\operatorname{trp} D$ and $\operatorname{trpE}$ for these strains and there are no other regions of these draft genomes that appear to encode phage-derived bacteriocins. This genomic context matches to that found in strain UB246, which, combined with their phylogenetic placement (Fig. 1), suggests that this may be the ancestral context for this region in $P$. syringae. Therefore, the ability to produce phage-derived bacteriocins from a locus in between trpD/trpE has either been lost or has never been acquired by this group.

Conversely, although many closely related strains that belong to phylogroups I (4 of 10 strains) and III (4 of 7 strains) do not appear to produce R-type syringacins targeting strains screened for sensitivity in this study, these strains do possess some structural genes for R-type syringacin production. These strains have at least a semblance of the genetic potential to create phage-derived bacteriocins but either lack the ability to make a subset of required proteins or target a limited number of strains that are not represented in our screen. In some cases, we have previously noted instances where genomes appear to lack crucial proteins for the production of these molecules and it is likely that these truly are nulls [14]. However, we also note that genome sequences for strains suggest that there are mutations that truncate critically important proteins (i.e., in $P s v$, the chaperone protein has an early stop codon [data not shown]).

\section{Localized recombination involving the $\mathrm{Rbp}$ and chaperone drive changes in R-type syringacin killing spectra}

Even though R-type syringacins are composed of multiple proteins, we find that diversity in targeting capability can at least partially be explained by changes in a pair of genes that encode an Rbp and its chaperone, the former being critical for target cell recognition. Moreover, we have demonstrated that acquisition of just these two genes can lead to changes in targeting capability for syringacins by expressing the Pja pair in a strain of PsyB728a devoid of its native $\mathrm{Rbp}$ and chaperone. Similarly, we have found that specific targeting is transferable with the Rbp and chaperone pair from Pae and Pmo (data not shown). While recombination is known to occur within $P$. syringae, recombination events are usually dominated by events between strains within the same phylogroup [28, 29]. Given sampling biases inherent in these data, it is difficult to make quantitative statements on rates of recombination. However, comparison of phylogenies within Fig. 3, and specifically the multiple cases where strains from different phylogroups share closely related Rbp alleles, suggests that recombination events can occur broadly across phylogroups. Moreover, further comparison suggests that recombination events involving these loci can also occur within phylogroups (i.e., Rbp of Psy301D). Taken together, patterns in both the frequency and the nature of recombination strongly support the idea that evolutionary dynamics of the Rbp and chaperone loci differ from much of the rest of the core genome.

$P$. syringae genomes are highly plastic in terms of gene presence and absence, but acquisition of loci through horizontal gene transfer is thought to occur mainly through phage transduction or conjugation [25, 29]. It is difficult to imagine how such high levels of site-specific recombination can be achieved through either conjugation or transduction across these strains although it is possible that single-strand DNA (ssDNA) containing these loci could be packaged and transferred along with other phage [30]. To this point, production of ssDNA has been witnessed from R-type 
pyocin loci but the ability of such particles to be packaged and transferred remains unexplored. Localized recombination with clean replacement of extant alleles, as seen with the Rbp and chaperone loci, is more likely to occur through natural transformation of extracellular DNA followed by homologous recombination rather than site-specific recombination. While there is one report of natural transformation in P. syringae occurring in planta [31], strains have typically been recalcitrant to recombination of extracellular DNA under laboratory conditions and the signals that trigger competence for natural transformation remain undefined. Transformation may also be mediated through the production of vesicles [32]. At present given the weight of all data, although these recombination patterns are easily explained by natural transformation of $P$. syringae, exact mechanisms enabling such localized recombination across $P$. syringae strains remain unclear. An alternative explanation for the high frequency of recombination between Rbp/ chaperone pairs among $P$. syringae is that the exchange is phage mediated, but not general or special transduction per se, but that the Rbp/chaperone pairs are shared with extant phages. In this scenario, phages (likely Myoviridae) would utilize the same Rbp/chaperone to infect $P$. syringae hosts, but rather than leading to replication and lysis or being maintained as prophages, the infecting phage recombines with the R-type syringacin. The promiscuity of phages isolated from phyllosphere environments, including those able to infect $P$. syringae, suggests that this is a reasonable hypothesis [33].

\section{Overall patterns in P. syringae killing activity}

Although sampled strains were biased toward the wellstudied phylogroups I, II, and III, our screen included representatives from 9 of the 13 acknowledged phylogroups [26]. One major trend that stands out is that phylogroup II strains appear to possess an above average tendency to be able to kill other strains, while phylogroup I and III strains contain an overabundance of "non-producers". While it remains a possibility that this pattern is due solely to sampling bias, and given the caveat that "non-producers" may just have a more limited target range, we have included a minimum of 8 strains from each phylogroup reflecting overall evolutionary diversity and phytopathogenic host range of these groups. It may be a coincidence, but strains from phylogroup II also tend to be isolated more frequently from a variety of environmental sources and are also pathogenic on a more diverse suite of hosts than other phylogroups [26]. It will be interesting for future studies to address how such capabilities are reflective of ecological niches. Previous studies with R-type pyocins and other bacteriocins have strongly suggested that these molecules are widely used to outcompete other strains under natural conditions $[13,34]$. With this idea in mind, the complete lack of R-type syringacins for phylogroup IX strains and to a more limited extent the lack of R-type syringacin activity from other strains also potentially reflects fundamental differences in ecological strategies compared to other $P$. syringae strains. To this point, phylogroup IX strains are potentially ecologically unique from other phylogroups because they have only been isolated from aquatic environments, not plant hosts [26].

\section{Subtle differences within killing spectra and sensitivity classes}

R-type syringacin killing spectra display qualitative differences across strains. However, within these broadly defined classes, it is apparent that many strains have similar, yet non-identical killing spectra and thus subtly differ in their overall pattern. These phenotypic differences may reflect amino acid differences between $\mathrm{Rbp}$ and chaperone pairs across strains, which could render R-type syringacins ineffective against a subset of hosts. It is also possible that there are genes outside of those known to function in targeting, where sequence diversity could alter the ability to attach to target cells or may otherwise affect the efficiency of killing. If this is the case, Rbp-chaperone pairs with identical sequences may differ in targeting spectra depending on the strain genomic background. To this point, we highlight strains CC1466 and CC1557 that have identical protein sequences for both their Rbps and chaperones but differ in killing spectra (Figs. 1 and 3a).

In terms of sensitivity, there are multiple strains that have convergently evolved to be sensitive to the same suite of syringacins. We have recently demonstrated that the main target of syringacins is the LPS, much like R-type pyocins [21, 22]. Numerous studies have investigated LPS diversity across $P$. syringae, as this structure is the target for many antibodies used to serotype strains before the advent of widespread sequence-based identification schemes $[35,36]$. These serotyping studies suggested that LPS structures are diverse and changeable and that strains within the species could be split into at least 15 different groups [35]. If LPS is the target of these Rtype syringacins, it is thus likely that single types of phage-derived bacteriocins can target multiple types of LPS found within P. syringae. Alternatively, it is possible that LPS structures have converged across strains in specific ways that enable similar sensitivities despite overall variability. This prediction arises from our observations that an R-type syringacin produced by one strain is often not only able to differentially target strains within the same pathovar but can also kill strains from different phylogroups and pathovars. However, previous serotyping studies often showed that pathovars were often 
consistently grouped into the same serotype by LPS pattern and that different pathovars were often grouped into different serotypes [35]. From an evolutionary perspective, convergence or diversification of LPS structures could be important when considering trade-offs to syringacin resistance, because regions of the LPS are known to be recognized as a microbe-associated molecular pattern by plant immune responses and thus changes in the LPS could directly affect virulence in planta [37]. Likewise, inherent LPS structures likely place constraints on which types of syringacin loci are able to be acquired by strains because self-targeting could occur if the "wrong" alleles of Rbps and chaperones are introduced to strains through recombination. The dangers of similar evolutionary tradeoffs were clearly demonstrated in a strain of $P$. aeruginosa evolved to form better biofilms, which changed LPS and left strains open to self-killing by pyocins [38].

\section{Caveats}

We have presented a large-scale screen of general bacteriocin activity across diverse $P$. syringae strains, followed by selection of R-type syringacin activity from these same samples. Although we are confident that much of the activity is as reported, there remain a couple of caveats for these results. First, we note that lysogenic bacteriophage will likely be induced by the same mitomycin C treatments as bacteriocins [39]. While we already discounted phage activity wherever single plaques were apparent during the overlay assays, it is possible that some of the reported activity is due to phage that are at high enough concentrations that single plaques blend together and therefore converge upon bacteriocin killing phenotypes. If we have scored phage activity as bacteriocin activity, this will lead to an overestimation of bacteriocin killing; however, we remain confident given overall patterns that a far majority of the activity reported is due to bacteriocins.

Weak killing activity in any of the assays is challenging to score, and it is possible that very weak activity (due to a low number of killing particles or due to overgrowth by the overlay strain) could be scored as a null in our assays. To counter this, we have repeated all inductions, PEG selections, and overlays at least twice independently and have reported results where only one of the two assays showed activity. We have found that tailocin particles are concentrated during PEG precipitation, and thus it is unlikely that lack of observed activity after PEG selection is the product of loss of tailocin particles in the far majority of cases. We also note that, as with any selection, recovery of killing particles after PEG treatment is based upon assumptions of the biophysical properties of the particles themselves. For instance, strain CC1543 appears to have a killing spectrum largely consistent with tailocin activity from other strains, but this activity does not precipitate with PEG. It is possible that this strain does harbor an active tailocin, but for currently unknown reasons (i.e., perhaps a shorter tail), this activity does not precipitate with PEG.

\section{Conclusions}

We have demonstrated that phage-derived bacteriocin killing activities can be grouped into multiple clusters, and yet within each cluster there exists subtle differences from strain to strain in terms of activity. Furthermore, sensitivity to phage-derived bacteriocins across strains can also be grouped into multiple clusters, the composition of which is highly correlated with each strain's killing activity. We have shown that killing spectra for phage-derived bacteriocins can change rapidly between closely related strains and that localized recombination of just two genes appears to be sufficient to explain some transitions between killing groups. Even though recombination is known to occur within $P$. syringae, typically it is thought to occur between strains within phylogroups. However, some of the recombination events witnessed here inherently must have occurred between recipient and donor strains from different phylogroups. Switches between killing and sensitivity also evolve frequently between strains and could speak to larger selective pressures under natural conditions.

It is possible that the interactions reported in this manuscript are highly relevant to understanding structure within natural communities of $P$. syringae. Given the strong selective pressures of bacteriocin killing, it will be interesting to investigate how both killing and sensitivity differ throughout space and time and across different communities on different host plants. In the very least, our data provide a blueprint for evaluating expectations for intermicrobial interactions driven by phage-derived bacteriocins within species.

\section{Materials and methods}

\section{Strains and growth conditions}

Genome sequences for all wild-type $P$. syringae strains used in this study are publicly available, and assemblies can be found listed in https://doi.org/10.6084/m9.figshare. 5738523. Strains and plasmids constructed for experiments performed in this study are listed in Table 1. Forbasic maintenance and propagation, all strains were grown on King's B media (KB) unsupplemented with 
Table 1 Strains and plasmids constructed for this study

\begin{tabular}{|c|c|c|c|c|}
\hline \multicolumn{2}{|l|}{ Strain } & \multicolumn{2}{|l|}{ Description } & Reference \\
\hline \multicolumn{2}{|l|}{ Psy B278a } & \multicolumn{3}{|l|}{ P. syringae pv. syringae $\mathrm{B} 278 \mathrm{a}$} \\
\hline \multicolumn{2}{|l|}{ Pja } & \multicolumn{3}{|l|}{ P. syringae pv. japonica MAFF301072 } \\
\hline \multicolumn{2}{|c|}{ PsyB728a $\Delta$ Rrbp/KLH5 } & \multicolumn{2}{|c|}{$\begin{array}{l}\text { Psy B728a with clean deletion of Rbp and chaperone } \\
\text { (psyr_4584 and psyr_4585) }\end{array}$} & {$[14]$} \\
\hline \multicolumn{2}{|c|}{$\begin{array}{l}\text { PsyB728a } \Delta \text { Rrbp+pDAB296/ } \\
\text { KLH24 }\end{array}$} & \multicolumn{2}{|c|}{$\begin{array}{l}\text { PsyB } 728 \text { a } \Delta \text { Rrbp complemented with PsyB } 728 \text { a Rbp and } \\
\text { chaperone }\end{array}$} & {$[14]$} \\
\hline \multicolumn{2}{|c|}{$\begin{array}{l}\text { PsyB728a } \Delta \text { Rrbp }+ \text { pRbp-B728a/ } \\
\text { KLH27 }\end{array}$} & \multicolumn{2}{|c|}{$\begin{array}{l}\text { PsyB } 728 \text { a } \Delta \text { Rrbp complemented with PsyB } 728 \text { a Rbp and } \\
\text { chaperone }\end{array}$} & {$[14]$} \\
\hline \multicolumn{2}{|c|}{$\begin{array}{l}\text { PsyB728a } \Delta \text { Rrbp+pRbp-Pjal } \\
\text { KLH41 }\end{array}$} & \multicolumn{2}{|c|}{$\begin{array}{l}\text { PsyB728a } \Delta \text { Rrbp complemented with Pja Rbp and } \\
\text { chaperone }\end{array}$} & This paper \\
\hline Plasmid & Description & & Reference & \\
\hline pBV226 & Gateway destinati & on vector with expression driven by $n p t I I$, TetR & [44] & \\
\hline pDAB296 & MTN1970-2 EV c & construct in pBV226, TetR & {$[45]$} & \\
\hline pRbp-B728a & Rbp and chaperon & e ORFs from PsyB728a in pBV226, TetR & [14] & \\
\hline pRbp-Pja & Rbp and chaperon & ORFs from $P j a$ in $\mathrm{pBV} 226$, TetR & This paper & \\
\hline
\end{tabular}

antibiotics and incubated at $27^{\circ} \mathrm{C}$. Where applicable, antibiotics were used at the following concentrations: tetracycline $10 \mu \mathrm{g} / \mathrm{mL}$, rifampicin $50 \mu \mathrm{g} / \mathrm{mL}$, nitrofurantoin $40 \mu \mathrm{g} / \mathrm{mL}$.

\section{Bacteriocin induction and preparation}

The basic protocol for overlay assays has been described previously [40]. Briefly, for bacteriocin production, a single colony of the strain of interest is picked to $2-3 \mathrm{~mL} \mathrm{~KB}$ and grown overnight in a shaking incubator at $220 \mathrm{rpm}$ and $27^{\circ} \mathrm{C}$. The next day, strains were back diluted $1: 100$ in $3 \mathrm{~mL}$ of $\mathrm{KB}$ media and grown for 3-4 h. At this point, mitomycin $\mathrm{C}$ was added at $0.5 \mu \mathrm{g} / \mathrm{ml}$ concentration to induce bacteriocin production and cultures were incubated with shaking overnight. After this point, cultures were centrifuged at 20,000 $\times$ $g$ for $5 \mathrm{~min}$, and supernatant was taken and added to a new tube. To sterilize the supernatant, chloroform was added at $100 \mu \mathrm{l}$ per $1 \mathrm{~mL}$, vortexed for $15 \mathrm{~s}$, and incubated at room temperature for $1 \mathrm{~h}$. The supernatant was then centrifuged at $20,000 \times g$ for $5 \mathrm{~min}$. The upper aqueous layer was removed without any of the bottom chloroform layer, and then top layer was incubated in the fume hood for a minimum of $4 \mathrm{~h}$ to allow residual chloroform to evaporate.

\section{PEG precipitation}

Separation of high molecular weight R-type syringacins from the lower molecular weight bacteriocins compounds was performed by PEG precipitation. This was done by adding $\mathrm{NaCl}$ and PEG 8000 to final concentrations of $1 \mathrm{M}$ and $10 \%(\mathrm{w} / \mathrm{v}$ ), respectively. The supernatants were then left overnight at $4{ }^{\circ} \mathrm{C}$ or incubated for $1 \mathrm{~h}$ on ice. Centrifugation then took place for $30 \mathrm{~min}$ at $4{ }^{\circ} \mathrm{C}$. The supernatant was removed and the pellet was resuspended in 100-250 $\mu \mathrm{l}$ of buffer (10 mM Tris, $10 \mathrm{mM}$ MgSO4, pH 7.0). Residual PEG was removed by two sequential equal volume extractions with chloroform. Residual chloroform was allowed to evaporate from uncapped samples for $4 \mathrm{~h}$ in a fume hood.

\section{Soft agar overlay protocol}

A single colony of the strain being used as an overlay was picked to $2-3 \mathrm{~mL} \mathrm{~KB}$ and grown overnight in a shaking incubator at $220 \mathrm{rpm}$ and $27^{\circ} \mathrm{C}$. The next day, strains were back diluted 1:100 in $3 \mathrm{~mL}$ of $\mathrm{KB}$ media and grown for $3-4 \mathrm{~h}$. The $0.4 \%$ agar was melted and $3 \mathrm{ml}$ was added to a culture tube; once it had cooled down, $100 \mu \mathrm{l}$ of the strain of interest was added. It was then poured over a petri dish containing $\mathrm{KB}$ media and swirled to cover the entire dish. It was allowed to solidify for $15-20 \mathrm{~min}$ and then the $2 \mu \mathrm{l}$ of supernatant and PEG-prepped samples were spotted onto the dish. They were allowed to grow for 1-2 days and then the presence and absence of killing activity was recorded. All assays were performed twice, with two independent bacteriocin inductions (and subsequent PEG selection) and overlays per supernatant-overlay combination. A majority of the activity was repeatable across assays, but assays were repeated for a third time if results were inconsistent. If no clear result emerged after three assays (i.e., strong, weak, and no killing across these three assays), data were coded as the presence of "killing" but with a qualifier notation. Full killing data on interactions from across assays can be found here: https://doi.org/10.6084/m9.figshare.5734125. 


\section{Hierarchical clustering}

A smaller killing-sensitivity matrix was created using only the data from PEG-prepped supernatants, with the presence of killing activity coded as " 1 " and absence of activity coded as "0". In cases where activity was inconsistent across assays, data were coded as "1". All data on killing of Pla107 was excluded from this cluster analysis because of variability caused by a secondary killing activity due to megaplasmid present within this strain. The PEG-only killing matrix, sans Pla107, can be found here: https://doi.org/10. $6084 / \mathrm{m} 9$.figshare.5734122. Calculation of phenotypic distances between killing profiles for each strain was performed in $\mathrm{R}$ (version 3.3, ref. [41]) using the "dist" function and "euclidean" method. Hierarchical clustering was then performed on phenotypic distances using the "hclust" function and "ward.D2" method. R scripts used for these clustering calculations can be found here: https://doi.org/10.6084/m9. figshare.5738427. Profiles for sensitivity were calculated in the same way, except that data in the original PEG-only killing matrix was transposed. This transposed matrix can be found here: https://doi.org/10.6084/m9.figshare.5734119.

\section{Phylogenetics}

Nucleotide and protein sequences of all genes involved were identified through tblastn searches of available genomes. For multilocus sequence analysis (MLSA) phylogenies in Fig. 1, nucleotide sequences for fragments of gyrB, rpod, gltA, gapA, and $a c n B$ were concatenated and then aligned. For phylogenies in Fig. 3a, amino acid sequences for fragments of $\operatorname{TrpC} / \operatorname{TrpD}$ and tail sheath/tape measure protein were independently concatenated and aligned. Amino acid sequences of Rbps were aligned separately from the sequences. We have excluded the amino acid sequence of CC1583 from this analysis because it was truncated within the draft genome assembly and we could not identify the $\mathrm{C}$ terminus for this allele. In all cases, sequences were aligned using ClustalX [42] with default parameters, with alignments polished by hand if necessary. Alignments for all sequences used to infer phylogenies can be found at https://doi.org/10.6084/m9.figshare.5734128, https://doi.org/10.6084/m9.figshare.5734131, and https:// doi.org/10.6084/m9.figshare.5734134.

All phylogenies were inferred using MrBayes 3.0 [43]. For MLSA phylogenies, convergence occurred after 1,000,000 generations and a burn in of 250,000 was used. For TrpC/ TrpD, convergence occurred after 1,000,000 generations and a burn in of 250,000 was used. For tail sheath/tape measure protein phylogenies, convergence occurred after $1,500,000$ generations and a burn in of 375,000 was used. For Rbp phylogenies, convergence occurred after 2,500,000 generations and a burn in of 625,000 was used.
Retargeting of R-type syringacin killing activity

A construct (start codon to stop codon, see sequence at https://doi.org/10.6084/m9.figshare.5738436) containing the $\mathrm{Rbp}$ and its chaperone from $P$. syringae pv. japonica MAFF301072 was amplified from genomic DNA of this strain and recombined into pDONR207 using BP clonase (Invitrogen, Carlsbad, CA) to create plasmid pKLHECO24. pKLHECO24 was purified and the Rbp/chaperone construct was recombined into pBAV226 using LR recombinase (Invitrogen, Carlsbad, CA) to create pKLHECO26. The final plasmid, in which the Pja Rbp and chaperone are cotranscribed from an $n p t I I$ promoter, was mated into KLH5 ( $\Delta$ Rrbp from [14]), and a tetracycline-resistant colony was isolated. Overlays were performed as above, with all strains grown in unsupplemented $\mathrm{KB}$ media prior to induction. Whole pictures, cropped to create images for Fig. 4, are available here: https://doi.org/10.6084/m9.figshare.5734137.

Acknowledgements We thank Dr. Brian Kvitko and Brian Smith for extensive discussions about experiments and Ethan Carlson and Danny Plewa for laboratory assistance. We thank all researchers for access to strains but especially Dr. Cindy Morris at INRA Avignon. DAB and KLH are supported by US Department of Agriculture (USDA) NIFA 2016-67014-24805.

\section{Compliance with ethical standards}

Conflict of interest The authors declare that they have no conflict of interest.

Open Access This article is licensed under a Creative Commons Attribution 4.0 International License, which permits use, sharing, adaptation, distribution and reproduction in any medium or format, as long as you give appropriate credit to the original author(s) and the source, provide a link to the Creative Commons license, and indicate if changes were made. The images or other third party material in this article are included in the article's Creative Commons license, unless indicated otherwise in a credit line to the material. If material is not included in the article's Creative Commons license and your intended use is not permitted by statutory regulation or exceeds the permitted use, you will need to obtain permission directly from the copyright holder. To view a copy of this license, visit http://creativecommons. org/licenses/by/4.0/.

\section{References}

1. Koskella B, Hall LJ, Metcalf CJE. The microbiome beyond the horizon of ecological and evolutionary theory. Nat Ecol Evol. 2017;1:1606-15.

2. Coyte KZ, Schluter J, Foster KR. The ecology of the microbiome: networks, competition, and stability. Science. 2015;350:663-6.

3. Mueller UG, Sachs JL. Engineering microbiomes to improve plant and animal health. Trends Microbiol. 2015;23:606-17.

4. Sheth RU, Cabral V, Chen SP, Wang HH. Manipulating bacterial communities by in situ microbiome engineering. Trends Genet. 2016;32:189-200.

5. Bucci V, Nadell CD, Xavier JB. The evolution of bacteriocin production in bacterial biofilms. Am Nat. 2011;178:E162-73. 
6. Riley MA, Wertz JE. Bacteriocins: evolution, ecology, and application. Annu Rev Microbiol. 2002;56:117-37.

7. Hawlena H, Bashey F, Lively CM. Bacteriocin-mediated interactions within and between coexisting species. Ecol Evol. 2012;2:2521-6.

8. Andersson DI, Hughes D. Microbiological effects of sublethal levels of antibiotics. Nat Rev Microbiol. 2014;12:465-78.

9. Cotter PD, Ross RP, Hill C. Bacteriocins - a viable alternative to antibiotics? Nat Rev Microbiol. 2013;11:95-105.

10. Yang S-C, Lin C-H, Sung CT, Fang J-Y. Antibacterial activities of bacteriocins: application in foods and pharmaceuticals. Front Microbiol. 2014;5:241.

11. Gebhart D, Lok S, Clare S, Tomas M, Stares M, Scholl D. et al. A modified R-type bacteriocin specifically targeting Clostridium difficile prevents colonization of mice without affecting gut microbiota diversity. MBio. 2015;6:e02368-14

12. Williams SR, Gebhart D, Martin DW, Scholl D. Retargeting R-type pyocins to generate novel bactericidal protein complexes. Appl Environ Microbiol. 2008;74:3868-76.

13. Hert AP, Roberts PD, Momol MT, Minsavage GV, Tudor-Nelson SM, Jones JB. Relative importance of bacteriocin-like genes in antagonism of Xanthomonas perforans tomato race 3 to Xanthomonas euvesicatoria tomato race 1 strains. Appl Environ Microbiol. 2005;71:3581-8.

14. Hockett KL, Renner T, Baltrus DA. Independent co-option of a tailed bacteriophage into a killing complex in Pseudomonas. MBio. 2015;6:e00452.

15. Ghequire MGK, De Mot R. The tailocin tale: peeling off phage tails. Trends Microbiol. 2015;23:587-90.

16. Scholl D. Phage tail-like bacteriocins. Annu Rev Virol. 2017;4:453-67.

17. Taylor NMI, van Raaij MJ, Leiman PG. Contractile injection systems of bacteriophages and related systems. Mol Microbiol. 2018;108:6-15.

18. Gill JJ, Young R. (eds. Alita A. Miller and Paul F. Miller) Therapeutic applications of phage biology: history, practice and recommendations. In Emerging trends in antibacterial discovery: answering the call to arms. Caister Academic Press; Poole, U.K. 2011

19. Leiman PG, Shneider MM. Contractile tail machines of bacteriophages. Adv Exp Med Biol. 2012;726:93-114.

20. Matsui H, Sano Y, Ishihara H, Shinomiya T. Regulation of pyocin genes in Pseudomonas aeruginosa by positive (prtN) and negative (prtR) regulatory genes. J Bacteriol. 1993;175:1257-63.

21. Köhler T, Donner V, van Delden C. Lipopolysaccharide as shield and receptor for R-pyocin-mediated killing in Pseudomonas aeruginosa. J Bacteriol. 2010;192:1921-8.

22. Hockett KL, Clark M, Scott S, Baltrus DA. Conditionally redundant bacteriocin targeting by Pseudomonas syringae. bioRxiv. 2017. https://doi.org/10.1101/167593.

23. Vidaver AK, Buckner S. Typing of fluorescent phytopathogenic pseudomonads by bacteriocin production. Can J Microbiol. 1978;24:14-18.

24. Vidaver AK. Prospects for control of phytopathogenic bacteria by bacteriophages and bacteriocins. Annu Rev Phytopathol. 1976;14:451-65.

25. Baltrus DA, McCann HC, Guttman DS. Evolution, genomics and epidemiology of Pseudomonas syringae: challenges in bacterial molecular plant pathology. Mol Plant Pathol. 2017;18:152-68.

26. Berge O, Monteil CL, Bartoli C, Chandeysson C, Guilbaud C, Sands DC, et al. A user's guide to a data base of the diversity of Pseudomonas syringae and its application to classifying strains in this phylogenetic complex. PLoS ONE. 2014;9:e105547.

27. Vidaver AK, Mathys ML, Thomas ME, Schuster ML. Bacteriocins of the phytopathogens Pseudomonas syringae, $P$. glycinea, and $P$. phaseolicola. Can J Microbiol. 1972;18:705-13.
28. McCann HC, Li L, Liu Y, Li D, Pan H, Zhong C, et al. Origin and evolution of the Kiwifruit canker pandemic. Genome Biol Evol. 2017;9:932-44.

29. Dillion MM, Thakur S, Almeida RND, Guttman DS. Recombination of ecologically and evolutionarily significant loci maintains genetic cohesion in the Pseudomonas syringae species complex. bioRxiv. 2017. https://doi.org/10.1101/227413

30. Lee FK, Dudas KC, Hanson JA, Nelson MB, LoVerde PT, Apicella MA. The R-type pyocin of Pseudomonas aeruginosa C is a bacteriophage tail-like particle that contains single-stranded DNA. Infect Immun. 1999;67:717-25.

31. Lovell HC, Mansfield JW, Godfrey SAC, Jackson RW, Hancock JT, Arnold DL. Bacterial evolution by genomic island transfer occurs via DNA transformation in planta. Curr Biol. 2009;19:1586-90.

32. Renelli M, Matias V, Lo RY, Beveridge TJ. DNA-containing membrane vesicles of Pseudomonas aeruginosa PAO1 and their genetic transformation potential. Microbiology. 2004;150:2161-9.

33. Koskella B, Thompson JN, Preston GM, Buckling A. Local biotic environment shapes the spatial scale of bacteriophage adaptation to bacteria. Am Nat. 2011;177:440-51.

34. Lavermicocca P, Lonigro SL, Valerio F, Evidente A, Visconti A. Reduction of olive knot disease by a bacteriocin from Pseudomonas syringae pv. ciccaronei. Appl Environ Microbiol. 2002;68:1403-7.

35. Ovod V, Rudolph K, Krohn K. Serological classification of Pseudomonas syringae pathovars based on mononoclonal antibodies towards the lipopolysaccharide O-chains. Pseudomonas syringae pathovars and related pathogens. Dordrecht: Springer; 1997. p. 526-31.

36. Zdorovenko GM, Zdorovenko EL. Pseudomonas syringae lipopolysaccharides: Immunochemical characteristics and structure as a basis for strain classification. Microbiology. 2010;79:47-57.

37. Ranf S, Gisch N, Schäffer M, Illig T, Westphal L, Knirel YA, et al. A lectin S-domain receptor kinase mediates lipopolysaccharide sensing in Arabidopsis thaliana. Nat Immunol. 2015;16:426-33.

38. Penterman J, Nguyen D, Anderson E, Staudinger BJ, Greenberg EP, Lam JS, et al. Rapid evolution of culture-impaired bacteria during adaptation to biofilm growth. Cell Rep. 2014;6:293-300.

39. Lamont I, Brumby AM, Egan JB. UV induction of coliphage 186: prophage induction as an SOS function. Proc Natl Acad Sci USA. 1989;86:5492-6.

40. Hockett KL, Baltrus DA. Use of the soft-agar overlay technique to screen for bacterially produced inhibitory compounds. J Vis Exp. 2017:55064.

41. R Core Team. R: a language and environment for statistical. Vienna, Austria: R Foundation for Statistical Computing; 2014.

42. Thompson JD, Gibson TJ, Higgins DG. Multiple sequence alignment using ClustalW and ClustalX. Curr Protoc Bioinformatics. 2002; Chapter 2:Unit 2.3.

43. Ronquist F, Huelsenbeck JP. MrBayes 3: Bayesian phylogenetic inference under mixed models. Bioinformatics. 2003;19:1572-4.

44. Vinatzer BA1, Teitzel GM, Lee MW, Jelenska J, Hotton S, Fairfax K, Jenrette J, Greenberg JT. 2006 The type III effector repertoire of Pseudomonas syringae pv. syringae B728a and its role in survival and disease on host and non-host plants. Mol Microbiol. 2006 Oct;62:26-44.

45. Baltrus DA1, Nishimura MT, Dougherty KM, Biswas S, Mukhtar MS, Vicente J, Holub EB, Dangl JL. 2012 The molecular basis of host specialization in bean pathovars of Pseudomonas syringae. Mol Plant Microbe Interact. 2012 Jul;25(7):877-88. https://doi.org/10.1094/MPMI-08-11-0218. 